\title{
A comparative phylogenomic analysis of SARS-CoV-2 strains reported from non-human mammalian species and environmental samples
}

\author{
Aziz UI-Rahman 1,2,4 $\cdot$ Muhammad Abu Bakr Shabbir ${ }^{2} \cdot$ Muhammad Waqar Aziz $^{2} \cdot$ Saima Yaqub $^{2} \cdot$ Asif Mehmood $^{3}$. \\ Muhammad Asif Raza ${ }^{1}$ Muhammad Zubair Shabbir ${ }^{4}$
}

Received: 10 April 2020 / Accepted: 29 September 2020 / Published online: 26 October 2020

(c) Springer Nature B.V. 2020

\begin{abstract}
Coronaviruses (CoVs) infect a wide range of domestic and wild mammals. These viruses have a potential and tendency to cross-species barriers and infect humans. Novel human coronavirus 2019-nCoV (hCoV-19) emerged from Wuhan, China, and has caused a global pandemic. Genomic features of SARS-CoV-2 may attribute inter-species transmission and adaptation to a novel host, and therefore is imperative to explicate the evolutionary dynamics of the viral genome and its propensity for differential host selection. We conducted an in silico analysis of all the coding gene sequences of SARS-CoV-2 strains $(\mathrm{n}=$ 39) originating from a range of non-human mammalian species, including pangolin, bat, dog, cat, tiger, mink, mouse, and the environmental samples such as wastewater, air and surface samples from the door handle and seafood market. Compared to the reference SARS-CoV-2 strain (MN908947; Wuhan-Hu-1), phylogenetic and comparative residue analysis revealed the circulation of three variants, including hCoV-19 virus from humans and two hCoV-19-related precursors from bats and pangolins. A lack of obvious differences as well as a maximum genetic homology among dog-, cat-, tiger-, mink-, mouse-, bat- and pangolin-derived SARS-CoV-2 sequences suggested a likely evolution of these strains from a common ancestor. Several residue substitutions were observed in the receptor-binding domain (RBD) of the spike protein, concluding a promiscuous nature of the virus for host species where genomic alternations may be required for the adaptation to novel host/s. However, such speculation needs in vitro investigations to unleash the influence of substitutions towards species-jump and disease pathogenesis.
\end{abstract}

Keywords Novel coronavirus $\cdot$ SARS-CoV-2 $\cdot$ Non-human mammalian sequences $\cdot$ Phylogenomic analysis $\cdot$ Residue substitutions $\cdot$ Species-jump

Electronic supplementary material The online version of this article (https://doi.org/10.1007/s11033-020-05879-5) contains supplementary material, which is available to authorized users.

Aziz Ul-Rahman

drazizangel@gmail.com

1 Faculty of Veterinary and Animal Sciences, Muhammad Nawaz Shareef University of Agriculture, Multan 66000, Pakistan

2 Institute of Microbiology, University of Veterinary and Animal Sciences, Lahore 54600, Pakistan

3 Veterinary Research Institute, Zarar Shaheed road, Lahore 54000, Pakistan

4 Quality Operations Laboratory, University of Veterinary and Animal Sciences, Outfall road, Lahore 54600, Pakistan

\section{Introduction}

Coronaviruses (CoVs) have been identified from a diverse range of birds and mammals, including humans [1,2]. Most of the emerging CoVs, those have infected humans, are originated from animals or birds and have the potential to cause major outbreaks [2]. The CoVs have higher mutation rates and genetic variability in their genomes as a result of either recombination, genetic drift (point mutations), and lack of proofreading capacity by RNA-dependent polymerases (replicative fidelity and diversity) [3-6]. Indeed, the potential of $\mathrm{CoV}$ s for genetic variability has long been considered to be fundamental for their ever-lasting evolution, the emergence of new variants within a certain species, and even to new species resulting in epidemics and/or zoonoses. This phenomenon may simply be exemplified by the emergence of two zoonotic CoVs; the Severe Acute Respiratory Syndrome 
hCoV-19/Wuhan/IVDC-HB-envF13/2020|EPI ISL 408511

hCoV-19/Shanghai/SH0005/2020|EPI ISL 416319

hCoV-19/canine/Hong Kong/20-02756/2020|EPI ISL 414518

hCoV-19/USA/CA-CDPH-UC4/2020|EPI ISL 413561

hCoV-19/USA/WI-10/2020|EPI ISL 417204

hCoV-19/Beijing/235/2020|EPI ISL 413521

hCoV-19/USA/WAS109/2020|EPI ISL 417162

- hCoV-19/Beijing/BJ2456/2020|EPI ISL 430745

hCoV-19/Beijing/BJ2454/2020|EPI ISL 430744

hCoV-19/USA/WAS114/2020|EPI ISL 417167

hCoV-19/Singapore/4/2020|EPI ISL 410535

hCoV-19/NanChang/JX216/2020|EPI ISL 417420

hCoV-19/USA/CA9/2020|EPI ISL 412862

hCoV-19/Hangzhou/ZJU-01/2020|EPI ISL 415709

hCoV-19/Shandong/IVDC-SD-001/2020|EPI ISL 408482

- hCoV-19/mouse/Harbin/HRB-26m/2020|EPI ISL 459910

hCoV-19/Beijing/BJ2452/2020|EPI ISL 430743

hCoV-19/Italy/FVGICGEB S8/2020|EPI ISL 417421

hCoV-19/Wuhan/IVDC-HB-envF13-21/2020|EPI ISL 408515

hCoV-19/Germany/NRW-21/2020|EPI ISL 417466

hCoV-19/USA/MN47-MDH47/2020|EPI ISL 417494

hCoV-19/Italy/SPL1/2020 |EPI ISL 412974

- hCoV-19/canine/Hong Kong/20-03695/2020|EPI ISL 450403

hCoV-19/Wuhan/IVDC-HB-envF54/2020|EPI ISL 408512

hCoV-19/env/USA/MT-BWRF-0327/2020|EPI ISL 437434

MN908947 Wuhan-Hu-1

hCoV-19/Beijing/BJ2460/2020|EPI ISL 430746

42 hCoV-19/tiger/USA/NY-040420/2020|EPI ISL 420293

hCoV-19/Beijing/105/2020|EPI ISL 413518

hCoV-19/Spain/CastillayLeon201323/2020|EPI ISL 418248

hCoV-19/Portugal/PT0041/2020|EPI ISL 418026

hCoV-19/USA/WI-07/2020|EPI ISL 417201

hCoV-19/Canada/BC 41851/2020|EPI ISL 415584

hCoV-19/env/USA/FL-UF11/2020|EPI ISL 447900

hCoV-19/mink/Netherlands/NB02 16RS/2020|EPI ISL 447632

hCoV-19//taly/HSacco-1/2020|EPI ISL 429874

hCoV-19/mink/Netherlands/NB02 13KS/2020|EPI ISL 447630

hCoV-19/Italy/INMI6/2020|EPI ISL 419254

hCoV-19/Italy/INMI1cs/2020|EPI ISL 410546

- hCoV-19/Wuhan/IVDC-HB-envF13-20/2020|EPI ISL 408514

hCoV-19/Netherlands/NoordBrabant 52/2020|EPI ISL 415508

100

hCoV-19/USA/WA-UW70/2020|EPI ISL 415598

hCoV-19/England/09c/2020|EPI ISL 412116

hCoV-19/cat/France/53/2020|EPI ISL 437349

hCoV-19/env/USA/UN-UF-12/2020|EPI ISL 455682

hCoV-19/mink/Netherlands/NB02 index/2020|EPI ISL 447631

- hCoV-19/mink/Netherlands/NB02 07KS/2020|EPI ISL 447629

hCoV-19/mink/Netherlands/NB02 06KS/2020|EPI ISL 447628

hCoV-19/mink/Netherlands/NB04 index/2020|EPI ISL 447634

- hCoV-19/mink/Netherlands/NB03 index/2020|EPI ISL 447633

- hCoV-19/mink/Netherlands/NB01 04KS/2020|EPI ISL 447626

hCoV-19/mink/Netherlands/NB01 03KS/2020|EPI ISL 447625

hCoV-19/mink/Netherlands/NB01 02KS/2020|EPI ISL 447624

hCoV-19/mink/Netherlands/NB01 01KS/2020|EPI ISL 447623

hCoV-19/mink/Netherlands/1/2020|EPI ISL 431778

hCoV-19/env/USA/FL-UF3/2020|EPI ISL 434677

hCoV-19/mink/Netherlands/NB02 03KS/2020|EPI ISL 447627

hCoV-19/bat/Yunnan/RaTG13/2013|EPI ISL 402131

hCoV-19/pangolin/Guangxi/P5L/2017|EPI ISL 410540

hCoV-19/pangolin/Guangxi/P5E/2017|EPI ISL 410541

100

hCoV-19/pangolin/Guangxi/P1E/2017|EPI ISL 410539

hCoV-19/pangolin/Guangxi/P4L/2017|EPI ISL 410538

hCoV-19/pangolin/Guandong/1/2019|EPI ISL 410721

hCoV-19/pangolin/Guangxi/P2V/2017|EPI ISL 410542

hCoV-19/pangolin/China/MP789/2019|EPI ISL 412860

0.02 
४Fig. 1 Complete spike gene-based phylogenetic tree of SARS-CoV-2 strains reported from the human and non-human origin. Black circles indicate the sequences reported from mink (teal), environment (fuchsia), pangolin (blue), bat (red), dog (green), tiger (lime), cat (olive) and mouse (aqua). (Color figure online)

Coronavirus (SARS-CoV) and the Middle East Respiratory Syndrome Coronavirus (MERS-CoV) [2]. Since, it is believed that both CoVs have been transmitted to humans through intermediate mammalian host from a natural reservoir host (bats) [7, 8]. Therefore, bats are now considered as a potential reservoir host of CoVs that could be associated with future CoV-related pandemics [2].

Novel mammalian coronaviruses are now regularly identified [9-11]. Recently, a novel human coronavirus (hCoV-19) emerged in late December 2019 from Wuhan, China, and spread to more than 190 countries. This virus possesses a positive-sense, single-stranded RNA genome of approximately 30 kilobases in length. The genome encodes ten structural [ORFlab, Spike (S), Envelope (E), Membrane (M), Nucleocapsid (N), ORF3, ORF6, ORF7, ORF8, ORF10] proteins [12]. Though $S$ protein is a larger type-I membrane surface glycoprotein that plays an important role in tissue tropism and host adaptation [13, 14], other structural proteins are also reported to contribute significantly to the adaptation of CoVs to a novel host. For instance, a sequence of 29-nucleotides in the open reading frame (ORF10) could be considered an example that may play a role in the trans-species jump [15]. Deletion of the said sequence has been observed in most of the human-originated SARS-CoV strains; however, it has been reported vice versa for animal-originating strains. Similarly, insertion of a 29-nucleotide sequence in ORF8 protein of the bat-originating SARS-CoV strain was suggestive of a species-jump from bat to civet [15]. Although much of the genomic characteristics of SARS-CoV-2 are described so far; nevertheless, analysis revealing potential genetic properties underlying inter- and intra-species jump has largely remained elusive. With this background, we explored the genome of SARS-CoV-2 strains originating from a range of non-human mammalian species and environmental samples for the possible emergence of new variants and adaptation to a novel environment/host.

\section{Materials and methods}

To date (2-July 2020), a total of 51 genome sequences corresponding to novel human coronavirus (hCoV-19; SARS$\mathrm{CoV}-2$ ) are reported from non-human hosts and the environment at Global Initiative Sharing of All Influenza Data website (https://www.gisaid.org/). Only full-length nucleotide sequences were retrieved and screened for quality of sequences. The sequences with a long stretch of NNNs in coding genes were excluded from the phylogenetic, evolutionary, and comparative genomic analysis. Because of the greatest difference in spike protein, noises or recombination, or mixed reads of different viruses in metagenomic feces sample collected in 2019, two bat-derived hCoV-19 sequences (EPI ISL 412976 and EPI ISL 412977) were also excluded. Final dataset $(\mathrm{n}=39)$, analysed in this study, included SARS-CoV-2 strains originating from mink $(\mathrm{n}=$ 13), pangolin $(n=7), \operatorname{dog}(n=2)$, bat $(n=1)$, cat $(n=1)$, tiger $(n=1)$, mouse $(n=1)$ and environment including air, wastewater and surface samples $(\mathrm{n}=13)$. The human-originated SARS-CoV-2 sequence (NC_045512; MN908947; Wuhan-Hu-1) is considered as a reference sequence and retrieved from the GenBank public database (https://www. ncbi.nlm.nih.gov/). All coding gene sequences (ORFlab, S, E, M, N, ORF3a, ORF6, ORF7, ORF8, and ORF10) were individually aligned using the ClustalW method in BioEdit ${ }^{\circledR}$ version 5.0.6 [16]. To assess the phylogenetic relationship among human- and non-human-derived SARS-CoV-2 strains, a complete $\mathrm{S}$ gene-based phylogenetic tree was constructed using Maximum Likelihood (ML) statistical method of Tamura-Nei model with ML heuristic tree interference of Nearest Neighbor-Interchange (NNI) in MEGA ${ }^{\circledR}$ X [17], where reliability in the topology of the tree was assured with 1000 number of bootstrap replicas and $p$-distance substitution model. To determine the genomic divergence, a pairwise sequence comparison analysis was performed with the maximum composite likelihood method (d: transitions + transversions model) using all coding genes in MEGA ${ }^{\circledR}$ $X$ [17]. The rate and pattern of substitutions among sites were modeled with a gamma distribution (parameter $=1$ with homogenous lineage pattern). Moreover, a comparative residue analysis of the receptor-binding domain (RBD) (399-518 aa) at $\mathrm{S}$ protein was also performed to elucidate a presumptive role of substitutions in host adaptation.

\section{Results}

The phylogenetic analysis revealed a close relationship between SARS-CoV-2 strains originating from humans, dog, cat, tiger, mouse, mink, and environmental sources including air, surface, and wastewater. However, bat- and pangolinderived sequences clustered in separate clades. Noteworthy is the fact recently reported pangolin-derived sequences (2019) clustered closer to human-derived SARS-CoV-2 sequences than those reported in 2017 (Fig. 1). Compared to the human-originated SARS-CoV-2 sequence, pairwise residue similarity analysis of pangolin-derived SARSCoV-2 strains revealed maximum identity for spike protein $(90.15 \%)$ and ORFlab protein $(86.04 \%)$. However, for batderived hCoV-19, a maximum residue identity was found 
for ORF8 (94.78\%) and ORFlab (95.95\%) proteins. Among all the coding regions, E protein was conserved for all of the under-studied hCoV-19 sequences (Supplementary Table 1).

While comparing all structural proteins of reference hCoV-19 sequence, a maximum number of residue substitutions $(\mathrm{n}=971)$ was observed in pangolin-derived hCoV19 sequence followed by 313 substitutions in bat-derived hCoV-19 sequence and 7 substitutions each in dog- and wastewater-derived hCoV-19 sequences. A variable range of substitutions ( $\mathrm{n}=1-8)$ was observed in dog-, cat-, tiger-, mink-, mouse- and environment samples-derived hCoV-19 sequences (Table 1). Interestingly, there was an absolute lack of substitution in structural proteins of SARS-CoV-2 strains originating from the environment sample of the seafood market, China. A 30-bp (10 aa) deletion was observed in each of the ORFlab and spike proteins of pangolin-derived sequence whereas, 12-bp (4 aa) deletion was observed in the spike protein of bat-derived sequence. Pangolin-derived hCoV-19 sequences had shared maximum similar substitutions $(\mathrm{n}=189)$ with other under-studied $\mathrm{hCoV}-19$ sequences followed by bat ( $R$. affinis)-derived $\mathrm{hCoV}-19$ sequence ( $\mathrm{n}$ $=173)$, bat $(R$. malayanus)-derived $\mathrm{hCoV}-19$ sequence $(\mathrm{n}$ $=115$ ), wastewater-derived hCoV-19 sequence from USA $(n=5)$, tiger-derived $h C o V-19$ sequence $(n=4)$, air- and dog-derived hCoV-19 sequences ( $\mathrm{n}=3$ in each), cat- and door handle-derived $\mathrm{hCoV}-19$ sequences $(\mathrm{n}=2$ in each) and mink-, mouse and wastewater-derived hCoV sequences from Italy and China ( $\mathrm{n}=1$ in each) (Table 1$)$.

While comparing critical binding residues $\left({ }^{455} \mathrm{~L},{ }^{486} \mathrm{~F}\right.$, ${ }^{493} \mathrm{Q},{ }^{494} \mathrm{~S},{ }^{501} \mathrm{~N}$, and ${ }^{505} \mathrm{Y}$ ) in the receptor-binding domain (RBD) of the spike protein of human-derived reference sequence, five substitutions $\left({ }^{486} \mathrm{~L},{ }^{493} \mathrm{Y},{ }^{494} \mathrm{R},{ }^{501} \mathrm{D}\right.$, and $\left.{ }^{505} \mathrm{H}\right)$ were observed in the bat-derived $\mathrm{hCoV}-19$ sequence. On the other hand, four substitutions $\left({ }^{486} \mathrm{~L},{ }^{493} \mathrm{E},{ }^{494} \mathrm{R}\right.$, and $\left.{ }^{501} \mathrm{~T}\right)$ were observed in pangolin-derived hCoV-19 sequences. Similarly, a range of substitutions was also observed for interacting residues (ACE2-RBD) among non-humanderived hCoV-19 sequences. Nine substitutions $\left({ }^{403} \mathrm{~K},{ }^{417} \mathrm{~V}\right.$, ${ }^{439} \mathrm{~V},{ }^{445} \mathrm{~T},{ }^{483} \mathrm{Q},{ }^{484} \mathrm{~V},{ }^{490} \mathrm{Y},{ }^{498} \mathrm{H}$ and ${ }^{204} \mathrm{~N}$ ) were observed in pangolin-derived hCoV-19, eight substitutions $\left({ }^{403} \mathrm{~T},{ }^{439} \mathrm{~K}\right.$, ${ }^{445} \mathrm{E},{ }^{449} \mathrm{~F},{ }^{483} \mathrm{Q},{ }^{484} \mathrm{~T},{ }^{490} \mathrm{Y}$ and ${ }^{498} \mathrm{Y}$ ) in bat-derived hCoV19 , and one each in mink- $\left({ }^{453} \mathrm{~F}\right)$ and mouse- $\left({ }^{498} \mathrm{H}\right)$ derived hCoV-19 sequences. All binding and contact residues for ACE2-RBD interaction were found conserved in all other under-studied hCoV-19 sequences (Table 2).

\section{Discussion}

The analysis revealed that except for bat- and pangolinderived sequences, non-human mammalian- and environment-derived SARS-CoV-2 strains were more identical to human-originated strain. A close relationship between cat-, dog-, tiger-, mouse-, mink- and human-derived SARSCoV-2 strains may reflect a plausible spill-over of the virus among a wide range of species while living in close vicinity. This is not surprising because there are shreds of evidence suggesting transmission of $\mathrm{CoV}$ s from one animal to another or humans living in companionship and/or vicinity [18-20], and this is particularly true for pets and animals (e.g., Tiger) kept in captivity such as Zoo. It is now speculated that dogs and cats can be infected presumably after contracting the virus from infected pet-owners. Similarly, the tiger was found to have contracted the virus, likely from an infected but asymptomatic zookeeper. Either having a history of clinical or subclinical infection, the detection of SARS-CoV-2 genome in pets or animals living with and/or close vicinity to humans suggests human-to-animal transmission. Nevertheless, there does not exist or reported evidence for the potential animal-to-human transmission of the SARS-CoV-2 virus so far. Hence, this particular aspect needs further investigations that can explore the identification of biological or other relevant factors for the presence of virus in non-human mammalian hosts.

Given the phylogenetic pattern, the study suggests batand pangolin-derived sequences as prototypes or ancestral variants of the SARS-CoV-2 virus. Such observations highlight the evolutionary dynamics of the SARS-CoV-2 virus for the potential emergence of multiple variants or escape mutants as has significantly been evidenced for other CoVs [3, 21, 22]. Pangolin-, bat- and human-derived SARS-CoV-2 strains were distinct or distantly related in the phylogenetic analysis. However, as for the genetic divergence is concerned, bat- and human-derived SARS-CoV-2 strains were closer than pangolin-derived SARS-CoV-2 strains, indicating the emergence of variants from a common ancestor. Based upon genomic heterogeneity and clustering pattern, the study findings are in agreement with previous observations, suggesting a non-mosaic nature of the SARS-CoV-2 and its close relationship with bat-derived strain [12]. The analysis suggests that SARS-CoV-2 is not an exact variant which is causing a global pandemic in humans; however, the hypothesis of the emergence of the new variant from bats-derived CoVs is very likely. However, a controversy exists for a conceivable relationship among bats- and pangolin-derived hCoV-19 to human-originating SARSCoV-2 strains. Instead of direct transmission from bats to humans, a study suggests the role of another animal as an intermediate host of the SARS-CoV-2 virus [23]. Taking into consideration the recent history of emerged CoVs (e.g., SARS-CoV and MERS-CoV) where bat acted as a natural reservoir, while other animals (e.g., civet and camels) served as intermediate hosts before their transmission to terminal host (human) [7, 8], it seems reasonable to speculate that there may involve an intermediate host other than bats for potential transmission of SARS-CoV-2 to human. This is 


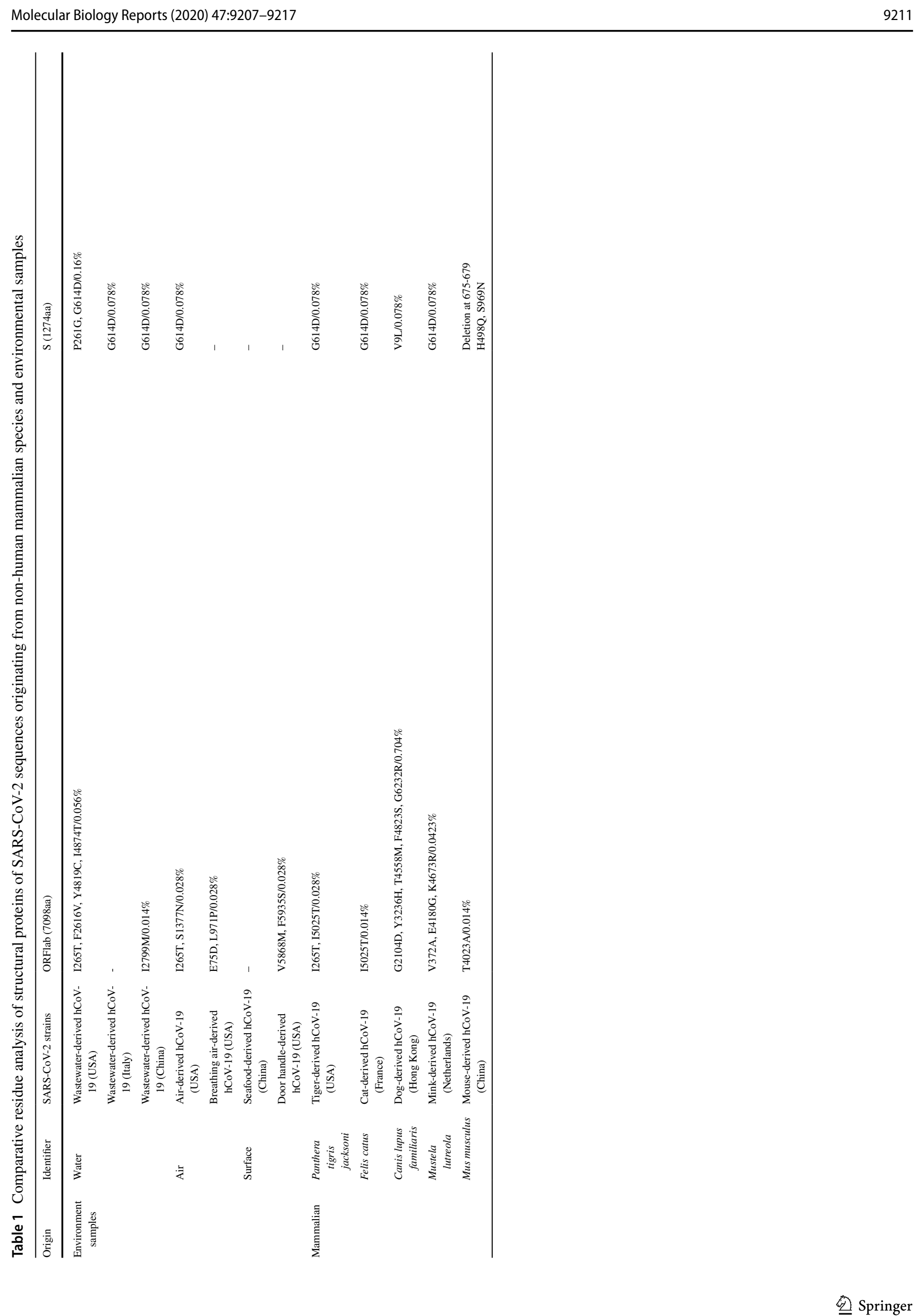




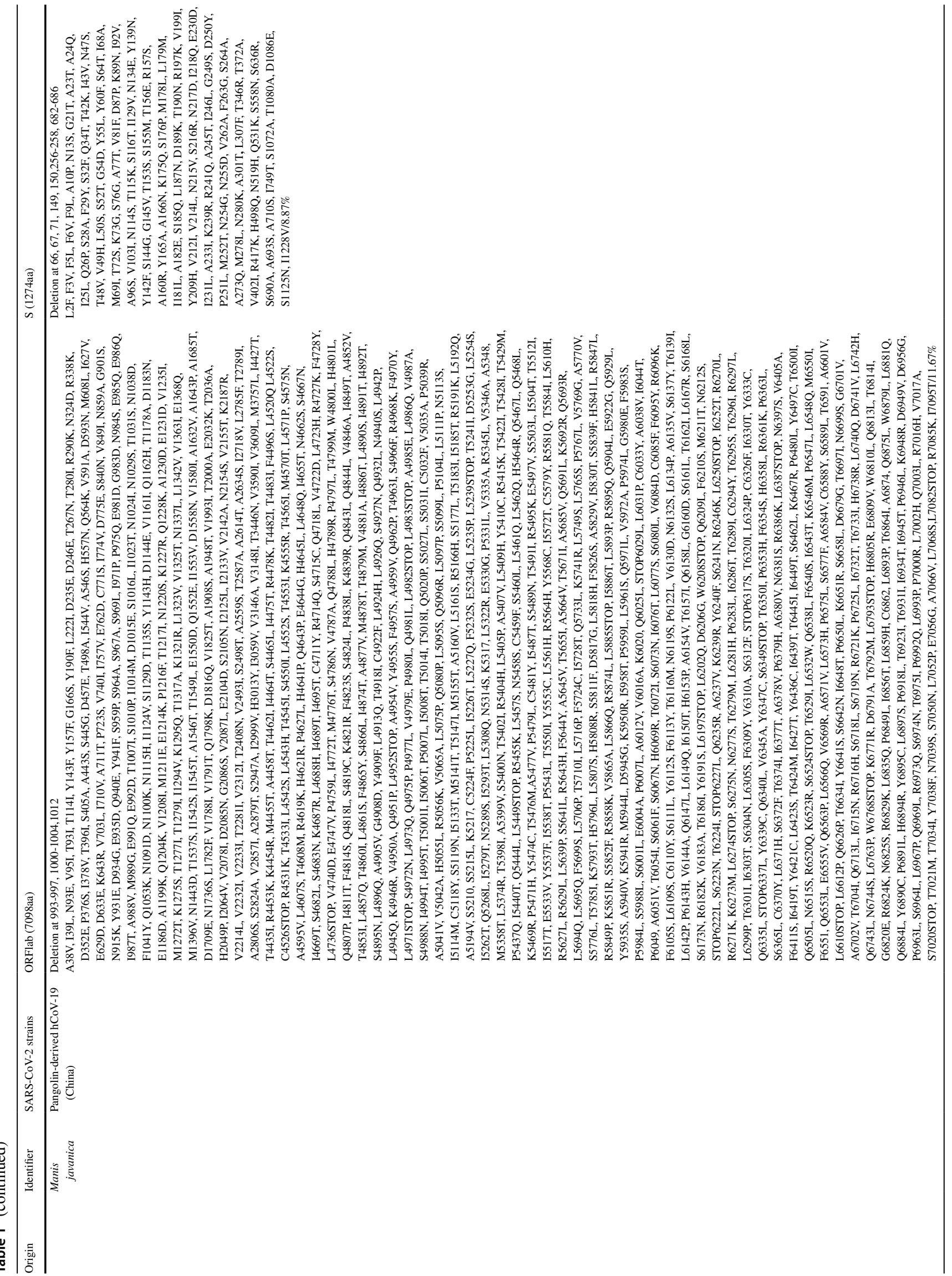




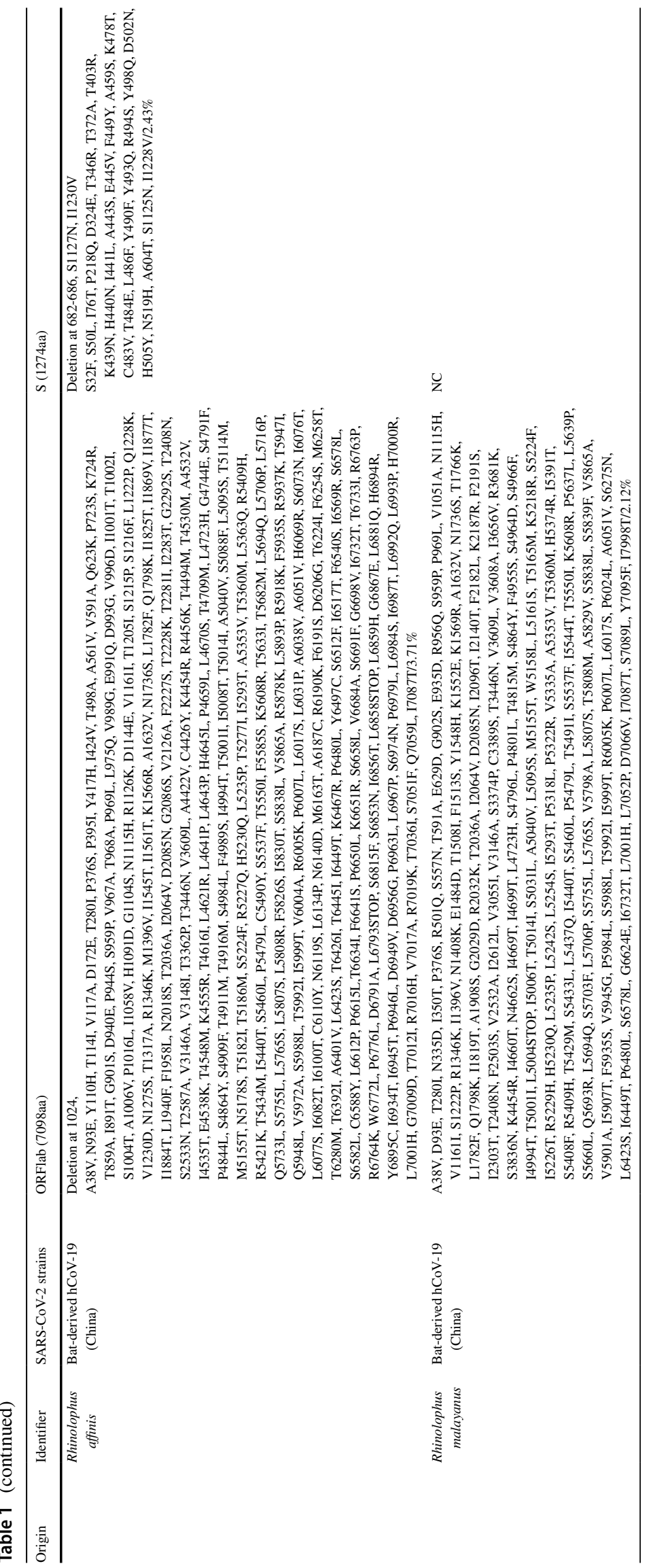




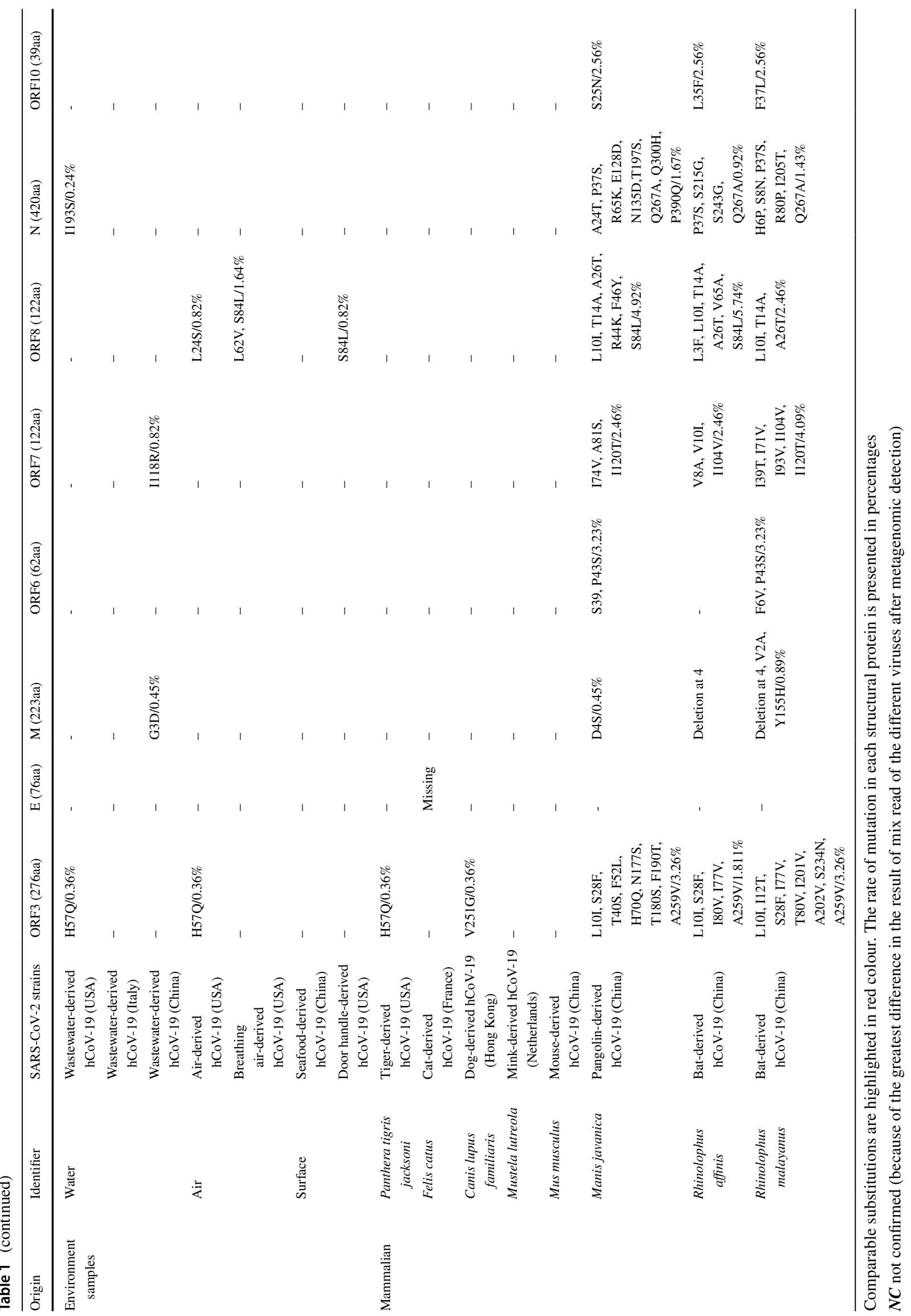




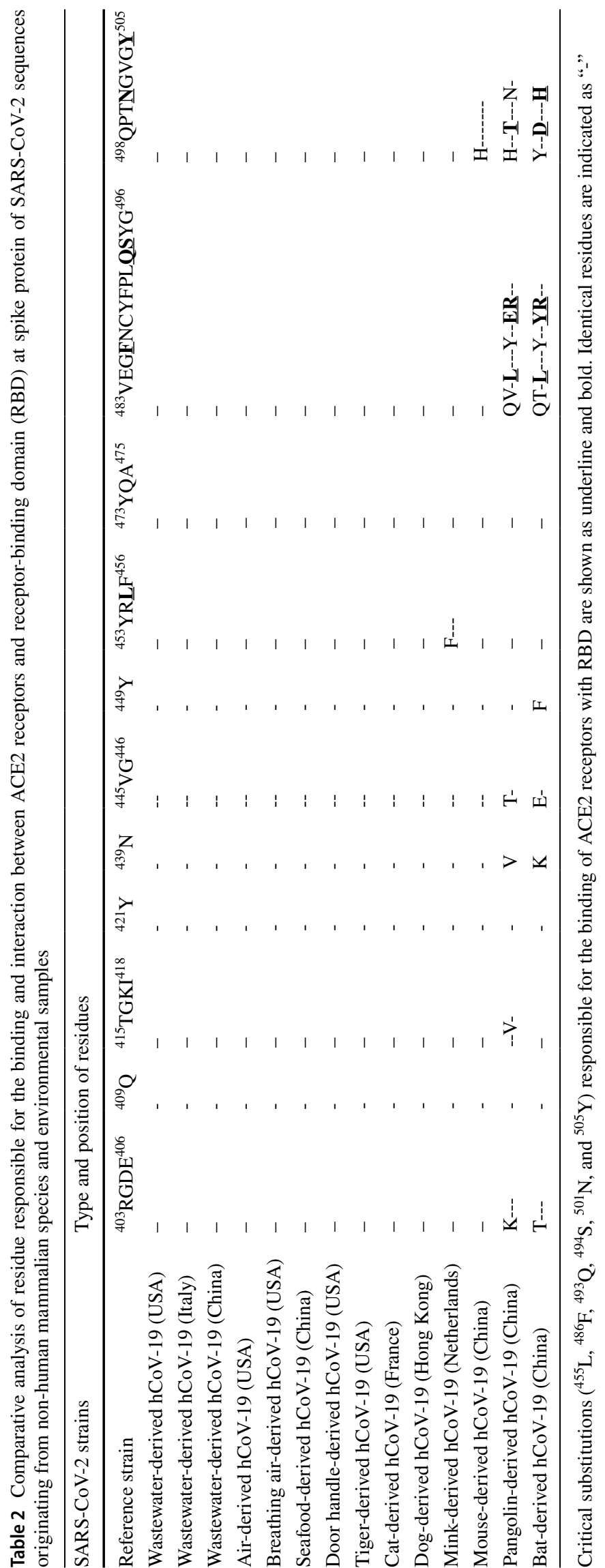


important because the place where the outbreak was first escalated during December 2019, most of the bats usually kept themselves in hibernation at that time of the year. Added to this, there was no selling of bat at the Huanan seafood market, whereas other non-aquatic animals such as pangolins were available. Therefore, this particular aspect needs further epidemiological investigations.

Such supposition is also linked to the detection of SARSlike coronavirus in pangolins [24]. On the other hand, the genetic makeup and similarity between spike protein of pangolin- and human-derived SARS-CoV-2 strains is higher $(90.15 \%)$ than what $(99 \%)$ is observed between humanand civets-derived SARS-CoV strains, which is why civets were considered as intermediate host but such scenario is not applicable in case of pangolin as intermediate host of SARS-CoV-2 virus. Regarding this aspect, the current study pointed out that pet animals including dogs and cats may act as an intermediate or carrier host of SARS-CoV-2 in the future because of a high level of genomic similarity between dog-, cat- and human-derived hCoVs sequences. This speculation is also supported by the reports about the pet animals as intermediate/carrier hosts of other human emerging viruses [25, 26]. However, it should be cautioned that such speculation is biased because of a limited number of dog- and cat-originating SARS-CoV-2 strains. Recent studies also claimed very low or permissive susceptibility of dogs and cats to SARS-CoV-2 infection [27] and found negative for the existence of SARS-CoV-2-specific antibodies [28]. There are still controversies about the source of the virus and its potential intermediate host which still needs more evidence to be confirmed. Therefore, it is quite earlier to conclude or proclaim any pet animal as an intermediate or carrier host of the SARS-CoV-2 virus and a slew of deep genomic analysis along with disease surveillance, sero-surveillance, molecular epidemiology, pathobiology, and transmissibility investigations has yet to find a conclusive proof.

An occurrence of residue substitutions in coding regions of SARS-CoV-2 sequences reported from different hosts highlight the potential of the virus to mutate rapidly for the emergence of host-specific variants. Considering SARSCoV-2 as a typical RNA virus, there is likely a chance of high mutation rate and subsequent speciation and evolution of novel variants. It is interesting to note that, among all the CoVs, SARS-CoV-2 possesses the longest genome that makes it more prone to errors during RNA transcription, and therefore the potential occurrence of mutations for the adaptation to novel hosts may very frequent. A significant number of residue substitutions were observed in RBD at spike protein of bat- and pangolin-derived SARS-CoV-2 strains. A vital role of mutations in the RBD region has been indicated for potential inter- and intra-species transmission of SARS-CoV [29]. Amino acid substitutions within the RBD can significantly affect the binding affinity of $S$ protein to its receptor. Any substitution at critical binding sites significantly lowers the electrostatic stabilizing interactions and binding affinity between ACE2-RBD and subsequently alter tissue tropism [30]. Indeed, a minimum of 1-2 substitutions in the RBD is sufficient for a virus to alter its host receptor specificity and subsequent inter-species transmission [31]. Beside it was reported that the SARS-CoV-2 virus has a unique peptide (PRRA) insertion at 680 amino acid position, which may be involved in the proteolytic cleavage of the $S$ protein by cellular proteases, and impact host range and transmissibility [32]. In the current comparative study, it is not found in the $\mathrm{S}$ proteins of the bat- and pangolin-derived SARS-CoV-2 strains. Comparative investigations to determine an in vitro impact of the mutations are prerequisites to explore species jump of the SARS-CoV-2 virus.

In conclusion, taken into consideration the intrinsic capacity of CoVs to adapt novel host species [33], the current study suggests the potential evolution of all SARS$\mathrm{CoV}-2$ strains from a common ancestor. Comparative analysis of human- and non-human-derived SARS-CoV-2 strains highlights the positive selection and continuous evolution in different hosts. Hence, the emergence of different variants with varying pathobiological characteristics is very likely for different host species living across the globe. In this regard, increased genetic diversity among SARS-CoV-2 infected patients suggests an adaptation as well as the evolution of the virus to the environment and the human population worldwide [34]. Therefore, an in-depth analysis of residue substitutions and genetic evolution of SARS-CoV-2 from a wide geographical region is ascertained in the future. Summarizing together, such comparative analysis enhances our understanding of the molecular epidemiology of the SARS$\mathrm{CoV}-2$ virus in a wide range of host species and future intervention strategies.

Acknowledgements The authors thank the anonymous reviewer for valuable and critical comments.

\section{Compliance with ethical standards}

Conflict of interest The authors declare that they have no conflict of interest.

Ethical approval This study does not contain any human or animal investigation

Informed consent No animal or human was involved

\section{References}

1. Tang XC, Zhang JX, Zhang SY, Wang P, Fan XH, Li LF, Li G, Dong BQ, Liu W, Cheung CL, Xu KM (2006) Prevalence and genetic diversity of coronaviruses in bats from China. J Virol 80(15):7481-90 
2. de Wit E, van Doremalen N, Falzarano D, Munster VJ (2016) SARS and MERS: recent insights into emerging coronaviruses. Nat Rev Microbiol 14(8):523

3. Su S, Wong G, Shi W, Liu J, Lai AC, Zhou J, Liu W, Bi Y, Gao GF (2016) Epidemiology, genetic recombination, and pathogenesis of coronaviruses. Trends Microbiol 24(6):490-502

4. Denison MR, Graham RL, Donaldson EF, Eckerle LD, Baric RS (2011) Coronaviruses: an RNA proofreading machine regulates replication fidelity and diversity. RNA Biol 8(2):270-279

5. Ren L, Zhang Y, Li J, Xiao Y, Zhang J, Wang Y, Chen L, Paranhos-Baccalà G, Wang J (2015) Genetic drift of human coronavirus OC43 spike gene during adaptive evolution. Sci Rep 22:511451

6. Zhang Y, Li J, Xiao Y, Zhang J, Wang Y, Chen L, ParanhosBaccalà G, Ren L, Wang J (2015) Genotype shift in human coronavirus OC43 and emergence of a novel genotype by natural recombination. J Infect 70(6):641-50

7. Ng SK (2003) Possible role of an animal vector in the SARS outbreak at Amoy Gardens. Lancet 362(9383):570-2

8. Hemida MG, Elmoslemany A, Al-Hizab F, Alnaeem A, Almathen F, Faye B, Chu DK, Perera RA, Peiris M (2017) Dromedary camels and the transmission of Middle East respiratory syndrome coronavirus (MERS-CoV). Transbound Emerg Dis 64(2):344-53

9. Woo PC, Lau SK, Lam CS, Lau CC, Tsang AK, Lau JH, Bai R, Teng JL, Tsang CC, Wang M, Zheng BJ (2012) Discovery of seven novel Mammalian and avian coronaviruses in the genus deltacoronavirus supports bat coronaviruses as the gene source of alphacoronavirus and betacoronavirus and avian coronaviruses as the gene source of gammacoronavirus and deltacoronavirus. $\mathrm{J}$ Virol 86(7):3995-4008

10. Poon LL, Chu DK, Chan KH, Wong OK, Ellis TM, Leung YH, Lau SK, Woo PC, Suen KY, Yuen KY, Guan Y (2005) Identification of a novel coronavirus in bats. J Virol 79(4):2001-9

11. To KK, Hung IF, Chan JF, Yuen KY (2013) From SARS coronavirus to novel animal and human coronaviruses. J Thoracic Dis 5(Suppl 2):S103

12. Lu R, Zhao X, Li J, Niu P, Yang B, Wu H, Wang W, Song H, Huang B, Zhu N, Bi Y (2020) Genomic characterization and epidemiology of 2019 novel coronavirus: implications for virus origins and receptor binding. Lancet 395(10224):565-74

13. Gallagher TM, Buchmeier MJ (2001) Coronavirus spike proteins in viral entry and pathogenesis. Virology 279(2):371-4

14. De Haan CA, Te Lintelo E, Li Z, Raaben M, Wurdinger T, Bosch BJ, Rottier PJ (2006) Cooperative involvement of the S1 and S2 subunits of the murine coronavirus spike protein in receptor binding and extended host range. J Virol 80(22):10909-18

15. Lu G, Wang Q, Gao GF (2015) Bat-to-human: spike features determining 'host jump'of coronaviruses SARS-CoV, MERS$\mathrm{CoV}$, and beyond. Trends Microbiol 23(8):468-78

16. Hall TA (1999) BioEdit: a user-friendly biological sequence alignment editor and analysis program for Windows 95/98/NT. In Nucleic Acids 135 Sympos Ser., 41, 95-98.

17. Kumar S, Stecher G, Li M, Knyaz C, Tamura K (2018) MEGA $\mathrm{X}$ : molecular evolutionary genetic analysis across computing platforms. Mol Biol Evol 35:1547-1549

18. Summa M, von Bonsdorff CH, Maunula L (2012) Pet dogsA transmission route for human noroviruses? J Clin Virol 53(3):244-7

19. Parrish CR, Murcia PR, Holmes EC (2015) Influenza virus reservoirs and intermediate hosts: dogs, horses, and new possibilities for influenza virus exposure of humans. J Virol 89(6):2990-4
20. Reusken CB, Raj VS, Koopmans MP, Haagmans BL (2016) Cross host transmission in the emergence of MERS coronavirus. Curr Opin Virol 16:55-62

21. Smits SL, Raj VS, Pas SD, Reusken CB, Mohran K, Farag EA, AlRomaihi HE, AlHajri MM, Haagmans BL, Koopmans MP (2015) Reliable typing of MERS-CoV variants with a small genome fragment. J Clin Virol 64:83-7

22. Lamers MM, Raj VS, Mahd Shafei SS, Abdallh SM, Gazo M, Nofal S, Lu X, Erdman DD, Koopmans MP, Abdallat M, Haddadin A (2016) Deletion variants of Middle East respiratory syndrome coronavirus from humans, Jordan, 2015. Emerg Infect Dis 22(4):716

23. Zhang T, Wu Q, Zhang Z (2020) Probable pangolin origin of SARS-CoV-2 associated with the COVID-19 outbreak. Curr Biol 30:1346-1351

24. Liu P, Chen W, Chen JP (2019) Viral metagenomics revealed sendai virus and coronavirus infection of malayan pangolins (Manis javanica). Viruses 11(11):979

25. Villabruna N, Koopmans MP, de Graaf M (2019) Animals as reservoir for human norovirus. Viruses 11(5):478

26. Zhang YZ, Fu ZF, Wang DM, Zhou JZ, Wang ZX, Lv TF, Xiong CL, Zou Y, Yao WR, Li MH, Dong GM (2008) Investigation of the role of healthy dogs as potential carriers of rabies virus. Vector-Borne Zoonotic Dis 8(3):313-320

27. Shi J, Wen Z, Zhong G, Yang H, Wang C, Huang B, Liu R, He X, Shuai L, Sun Z, Zhao Y (2020) Susceptibility of ferrets, cats, dogs, and other domesticated animals to SARS-coronavirus 2. Science. 368(6494):1016-20

28. Deng J, Jin Y, Liu Y, Sun J, Hao L, Bai J, Huang T, Lin D, Jin Y, Tian K (2020) Serological survey of SARS-CoV-2 for experimental, domestic, companion and wild animals excludes intermediate hosts of 35 different species of animals. Transbound Emerg Dis 2020(00):1-5. https://doi.org/10.1111/tbed.13577

29. Bolles M, Donaldson E, Baric R (2011) SARS-CoV and emergent coronaviruses: viral determinants of interspecies transmission. Curr Opin Virol 1(6):624-34

30. Li F (2013) Receptor recognition and cross-species infections of SARS coronavirus. Antiviral Res 100:246-54

31. Sheahan T, Rockx B, Donaldson E, Sims A, Pickles R, Corti D, Baric R (2008) Mechanisms of zoonotic severe acute respiratory syndrome coronavirus host range expansion in human airway epithelium. J Virol 82(5):2274-85

32. Li X, Zai J, Zhao Q, Nie Q, Li Y, Foley BT, Chaillon A (2020) Evolutionary history, potential intermediate animal host, and cross-species analyses of SARS-CoV-2. J Med Virol 92(6):602-11

33. Jo WK, Osterhaus AD, Ludlow M (2018) Transmission of morbilliviruses within and among marine mammal species. Curr Opin Virol 28:133-134

34. Shen Z, Xiao Y, Kang L, Ma W, Shi L, Zhang L, Zhou Z, Yang J, Zhong J, Yang D, Guo L, Zhang G, Li H, Xu Y, Chen M, Gao Z, Wang J, Ren L, Li M (2020) Genomic diversity of SARS-CoV-2 in coronavirus disease 2019 patients. Clin Infect Dis. https://doi. org/10.1093/cid/ciaa203

Publisher's Note Springer Nature remains neutral with regard to jurisdictional claims in published maps and institutional affiliations. 